\title{
The Effect of Bacteria on Epidermal Wound Healing
}

\author{
E. Agyingi * , S. Maggelakis and D. Ross \\ School of Mathematical Sciences, Rochester Institute of Technology \\ Rochester, NY 14623-5603, USA
}

\begin{abstract}
Epidermal wound healing is a complex process that repairs injured tissue. The complexity of this process increases when bacteria are present in a wound; the bacteria interaction determines whether infection sets in. Because of underlying physiological problems infected wounds do not follow the normal healing pattern. In this paper we present a mathematical model of the healing of both infected and uninfected wounds. At the core of our model is an account of the initiation of angiogenesis by macrophage-derived growth factors. We express the model as a system of reaction-diffusion equations, and we present results of computations for a version of the model with one spatial dimension.
\end{abstract}

Key words: wound healing, angiogenesis, growth factors, bacteria infection AMS subject classification: 92B05, 92C17, 92C 30, 92C 37

\section{Introduction}

Skin is the largest organ of the body. Its main function is to protect the internal organs. Skin damage, such as wounds caused by burns or cuts, constitutes serious injury and may lead to illness. Treatment of wounds has focused on how to close and repair the injured tissue rapidly. The healing of wounds is a complex process that involves various types of cells and chemical regulators. Wounding is an abnormal loss of cells. Healing begins when mitosis takes place to recover this loss, so the tissue mass is maintained in proper relation to body mass [3]. The healing process consists of three overlapping phases: the inflammation phase, in which the wound is prepared for healing, the proliferation phase, which is a re-constructive phase in which the dermal structure is

*Corresponding author. E-mail: eoasma@ rit.edu 
rebuilt, and the tissue remodeling phase, which supplies what is needed for the final form to be reached [5].

Injuries of epidermal tissue, such as cuts, cause disruption of blood vessels, which initiates the inflammation phase. During this phase, a wound is prepared for healing; the contaminants are removed, and neovascularization - the formation of new blood vessels begins. Growth of new blood vessels is necessary to supply the damaged tissue with blood, oxygen, and nutrients. The rate at which new blood vessels are generated affects the tissue repair process. The inflammatory stage comprises, in addition to neovascularization, the aggregation of platelets, which triggers coagulation and clotting. The clot shields the wound from further damage or infection and forms a matrix in which healing proceeds [6]. Later, macrophages migrate into the wound and secrete platelet-derived growth factors that mediate the inflammation part of the healing process [7]. If no blood is lost, platelets have no role in the healing process.

After inflammation is completed, re-growth begins. This is referred to as the proliferation phase. This is a rebuilding phase that is characterized by cell proliferation, cell migration, and the production of extracellular matrix, which is needed for the epidermal cells to migrate through collageneous dermis [8]. Neovascularization of the wound occurs through growth of blood vessels from areas adjacent to the wound [1]. Neovascularization is triggered by MDGFs [24]. During the proliferation stage a scar is formed. This is accomplished by a process called epithelialization in which the body produces collagen to strengthen the wound by supplying it with a thin protective coat that eventually thickens [20]. This covering prevents the wound from getting infected and retains wound fluid. Epithelialization is followed by wound contraction during which the sides of the wound are pulled together to shrink or close the wound area. Thus, the area to be restored by scar formation, for the wounds that are not closed, is reduced [26].

The last phase of wound healing is remodeling, which begins when the wound is closed. Wounds are closed before they are fully healed, that is, before the wounded bit of skin resumes normal functioning [21]. A wound may close without being fully healed. The ultimate goal is to return to normal function. In order for the wound to heal properly, remodeling of scar tissue must occur to allow the scar to change and fit the tissue. The process of scar remodeling is not thoroughly understood. Studies have suggested that the wound environment stimulates the fibroblast cells to synthesize and secrete a structural protein collagen, known as granulation tissue. Collagen is produced to hold the healing wound together, and it is a key ingredient of the healing process [20]. If no collagen is produced, the wound will not heal.

Although the phases involved in normal wound healing start sequentially, they overlap. A wound in which one of the above phases has been disrupted will not heal normally and may be infected. Infected wounds are often regarded as being stuck in the inflammation or proliferation phases of the healing process, and they contain bacteria whose interaction with the surrounding tissue determines the healing rate of the wound. A wound can contain bacteria without being infected. A wound that contains non-replicating bacteria is said to to contaminated. If the bacteria are replicating in the wound without cellular damage to the host, then the wound is said to be colonized. A wound becomes infected when replicating bacteria invade the tissues and causes damage. In an infected wound, the bacteria secrete exoenzymes that attack and destroy wound cells locally in order to obtained nutrients $[9,10]$. Infection can then rapidly spread throughout 
the wound. The progression from contamination to infection depends on the bacteria density, the virulence of the organisms and the host immune response. The healing process is slow compared with the spread of infection.

Mathematical models can shed light on the biological mechanisms of epidermal wound healing (see [17] for a review). We hope that modeling will lead to greater understanding of the process and improvements in wound care. Over the last two decades many mathematical models of aspects of wound healing have appeared in the research literature $[4,11,18,19,22,27]$. These models incorporate various crucial biological mechanisms such as cell mitosis, cell proliferation, cell death, capillary density, angiogenesis, oxygen supply, and production of growth factors. . Mathematical models of stall and infected wounds with focus on host tissue degradation by bacteria or quorum-sensing by bacteria have also been considered $[12,13,14,15,16,31]$. These papers provide complex models expressed as deterministic reaction-diffusion equations that must be solved numerically.

In this paper we present a multiscale model of the effects of micro organisms, such as bacteria in the wound, on the capillary remodeling. The model accounts for the dependence of capillary regeneration on the availability of oxygen concentration and the production of growth factors for non-contaminated and contaminated wounds. Models that focus on the global degradation of the host tissue by bacteria implicitly assume that bacteria have the ability to infect the wound. This is a limitation since wound contamination or colonization does not necessarily lead to an infection. It is known that a persistent production of endotoxin by bacteria leads to a decreased production of growth factors [25]. We attempt to capture how the presence of bacteria influences the production of growth factors. This approach allows us to investigate a matter that has been debated in the literature: whether infection is caused by bacterial density or bacterial virulence [2].

\section{The Mathematical Model}

We present a model of an epidermal wound in the presence of microbial organisms. In the interest of simplicity, we shall present our model as a spatially one-dimensional model. However, nothing about the model's structure demands that it be restricted to a single spatial dimension, and it can be generalized easily; one need only interpret the fluxes as higher-dimensional fluxes. The model accounts for the effect of oxygen, $O(x, t)$, on the production of the Macrophage-Derived Growth Factors (MDGFs), and the effect of the concentration of these growth factors, $G(x, t)$, on the increase of capillary density, $N(x, t)$, which is a combination of tip and sprout densities. The effect of bacteria density, $B(x, t)$, on the capillary remodeling is also examined. We let the wound occupy the interval $0 \leq x \leq R$, where the edges $x=0$ and $x=R$ denote the wound center and wound periphery respectively.

\subsection{Macrophage-Derived Growth Factor Model}

When the oxygen concentration is low, macrophages appear at the site of the wound. These macrophages release chemicals, MDGFs, that stimulate vessel growth and collagen deposition. 
The rate of production of MDGFs is a function of oxygen concentration. For our purposes we need only capture two elements of this function: its switch-like character, that is, the fact that it equals 0 for oxygen concentrations above a critical concentration, $\Theta$, and its decreasing monotonically with oxygen concentration. We take the MDFG production rate to be $\Phi(O)=(1-O / \Theta)$. Growth factor is taken up by capillaries at a rate that is proportional to both the capillary density and the growth factor concentration, $\gamma_{2} G N$. It is depleted in a second-order reaction with bacteria, at a rate $\gamma_{4} G B$. We account for natural loss of growth factor with a first-order loss term, $\gamma_{3} G$. The growth factor diffuses linearly in the wound region; we let $D_{1}$ be the diffusion coefficient. Thus, we have

$$
\frac{\partial G}{\partial t}=\nabla \cdot\left(D_{1} \nabla G\right)+\gamma_{1} \Phi(O)-\gamma_{2} G N-\gamma_{3} G-\gamma_{4} G B
$$

We assume that initially there is no growth factor: $G(x, 0)=0$. We also assume that there is no flux of growth factors through the boundaries. Thus we have the boundary conditions $G_{x}(0, t)=$ $G_{x}(R, t)=0$.

\subsection{Capillary Density Model}

The macrophage-derived growth factors (MDGFs) trigger the endothelial cells of the nearby blood vessels. This causes the growth of capillary tips. We model the increase in capillary density as having two parts: the increase, at a particular location, of the density to its natural limit, which we call $L$, and the spread of capillary tips from regions of greater to regions of lesser density.

If the capillary density were uniform in a region of the wound, it would increase until it reached its natural limit. We assume that this growth is logistic, that is, its rate is proportional to the capillary density and to the density of wound region still available for penetration by capillaries. At the MDGF concentrations that we consider, it is reasonable to assume that the growth rate of capillary density is also proportional to the concentration of MDGF. Thus we take this rate to be $\Psi(G, N)=G N(1-N / L)$.

Capillaries will also spread from regions of greater capillary density into regions of lesser capillary density. The mechanism for this spread is not really distinct from the mechanism we described in the prior paragraph. We model the spread of capillaries as mathematically analogous to nonlinear diffusion, although the capillaries do not diffuse, they grow. We take the "flux" of capillaries across a surface in the wound to be proportional to the rate of increase in capillary density described by $\Psi(G, N)$ and to the gradient of the capillary density. Thus this "flux" has the form $D_{2} \Psi(G, N) \nabla N$. By combining these two terms we obtain a PDE for the capillary density:

$$
\frac{\partial N}{\partial t}=\nabla \cdot\left(D_{2} \Psi(G, N) \nabla N\right)+\mu \Psi(G, N)
$$

We assume that initially $N(x, 0)=L$ at the edge $x=R$ and $N(x, 0)=0$ elsewhere. We set 
the flux of capillary density to zero at the boundary $x=0$ i.e. $N_{x}(0, t)=0$. We fix the capillary density at $x=R$ at the unwounded level, i.e. $N(R, t)=L$.

\subsection{Oxygen Transport Model}

Experimental results point out that the rate of wound healing depends on the oxygen supply. Increase of oxygen levels causes decrease in capillary growth. Thus, for successful healing to take place the oxygen level within the wound space must be low. The model assumes that the main source of oxygen is capillaries, and that it diffuses linearly $\nabla \cdot\left(D_{3} \nabla O\right)$ throughout the wound volume. We model oxygen production by the term $\lambda_{1} N$, and its depletion, which is as a result of uptake by the capillaries and linear decay by the term, $\lambda_{2} \mathrm{NO}-\lambda_{3}$. The equation governing oxygen production, transport and depletion is given by

$$
\frac{\partial O}{\partial t}=\nabla \cdot\left(D_{3} \nabla O\right)+\lambda_{1} N-\lambda_{2} O N-\lambda_{3} O .
$$

There is no oxygen flux at the center of the wound so that $O_{x}(0, t)=0$ and the concentration of oxygen at the other boundary is $O_{x}(R, t)=\theta$, which represents the oxygen density when there is no wound. We assume that oxygen density is nonzero only at the edge $x=R$ i.e. $O(x, 0)=\theta$, and $O(x, 0)=0$ elsewhere.

\subsection{Active Bacterial Density Model}

While intact skin provides an effective barrier to infection from extracellular bacteria, an open wound provides a favorable environment for bacteria. We assume that the wound is contaminated and that the bacteria survive on the substrate from the open wound. The available wound space $(1-N / L)$ therefore serves as the attractant concentration at spatial position $x$ and time $t$. We follow the Keller-Segel model of chemotaxis [29, 30], to model the growth and spread of the bacteria density in the wound volume. The following equation governs the bacteria density:

$$
\frac{\partial B}{\partial t}=\nabla \cdot\left(D_{4} \nabla B\right)-\nabla \cdot(\chi \nabla(1-N / L))+\alpha \Omega(N, B)
$$

where $\Omega(N, B)=(1-N / L) B(1-B / K)$ is the function describing bacteria growth, with rate $\alpha$ and carrying capacity $K$. The parameter $\chi$ is the chemotactic coefficient and $D_{4}$ is the bacteria diffusion coefficient. The initial bacterial distribution is given by $B(x, 0)=b_{0}$. We assume that there is no flux of bacteria through the boundaries. Thus we have the boundary conditions $B_{x}(0, t)=B_{x}(R, t)=0$. 


\subsection{Dimensionless Equations of the Model}

Setting $n=N / L, o=O / \theta, g=G / G_{0}$, (where $G_{0}$ is a characteristic MDGF concentration), and $b=B / K$, we obtain the following scale equations of the model:

$$
\begin{aligned}
& \frac{\partial n}{\partial \tau}=d_{1} \nabla \cdot(g n(1-n) \nabla n)+a_{1} g n(1-n) \\
& \frac{\partial o}{\partial \tau}=d_{2} \nabla \cdot(\nabla o)+a_{2} n-a_{3} o n-a_{4} O \\
& \frac{\partial g}{\partial \tau}=d_{3} \nabla \cdot(\nabla g)+a_{5}(1-o)-a_{6} g n-a_{7} g-a_{8} g b \\
& \frac{\partial b}{\partial \tau}=d_{4} \nabla \cdot(\nabla b)-\chi^{*} \nabla \cdot(\nabla(1-n))+a_{9}(1-n) b(1-b)
\end{aligned}
$$

where $x=R \xi, \quad t=T \tau, \quad d_{1}=D_{2} G_{0} L \epsilon, \quad d_{2}=D_{3} \epsilon, \quad d_{3}=D_{1} \epsilon, \quad d_{4}=D_{4} \epsilon, \quad a_{1}=$ $\mu G_{0} T, \quad a_{2}=\lambda_{1} L T / \theta, \quad a_{3}=\lambda_{2} L T, \quad a_{4}=\lambda_{3} T, \quad a_{5}=\gamma_{1} T / G_{0}, \quad a_{6}=\gamma_{2} L T, \quad a_{7}=\gamma_{3} T$, $a_{8}=\gamma_{4} K T, a_{9}=\alpha T, \quad \chi^{*}=\chi \epsilon / K$ and where $\epsilon=T / R^{2}$ for some characteristic time $T$. The initial conditions are $n(\xi, 0)=o(\xi, 0)=1$ at $\xi=1$ and $n(\xi, 0)=o(\xi, 0)=0$ elsewhere; $g(\xi, 0)=0$; and $b(\xi, 0)=b_{0} / K$. The boundary conditions are $n_{\xi}(0, \tau)=0$ and $n_{\xi}(1, \tau)=1$; $o_{\xi}(0, \tau)=0$ and $o_{\xi}(1, \tau)=1 ; g_{\xi}(0, \tau)=g_{\xi}(1, \tau)=0$; and $b_{\xi}(0, \tau)=b_{\xi}(1, \tau)=0$.

\section{Results and Discussion}

In this paper, we modeled the complex process of wound healing and bacteria contamination using a coupled system of nonlinear reaction-diffusion equations. We solve the non-dimensional equations numerically using the method of lines. The parameters used in our simulations were chosen to be in close proximity to similar parameters in related works $[18,23,27,28]$. The results can be summarized as the following three points:

First, we consider a wound that is free from bacteria contamination. All else being equal such a wound is expected to heal normally and this is demonstrated by the results in Figure 1 . We see a propagation of capillary density across the wound space at different times and this indicates that the wound is healing. It can be observed that where oxygen is low there is more production of MDGFs and this leads to the migration of capillaries. The regeneration of capillaries brings in more oxygen which then lowers the production of MDGFs. This is a negative feedback mechanism among oxygen, MDGFs, and capillaries. It is therefore clear that in the absence of any bacteria, the wound will heal successfully and in a timely manner.

As a second case, we consider wounds that are contaminated with non-virulent bacteria. Here, we present results for a wound whose bacteria density is low and another having a high bacteria density. The simulation in Figure 2 shows that when the bacteria density is low the healing rate of 

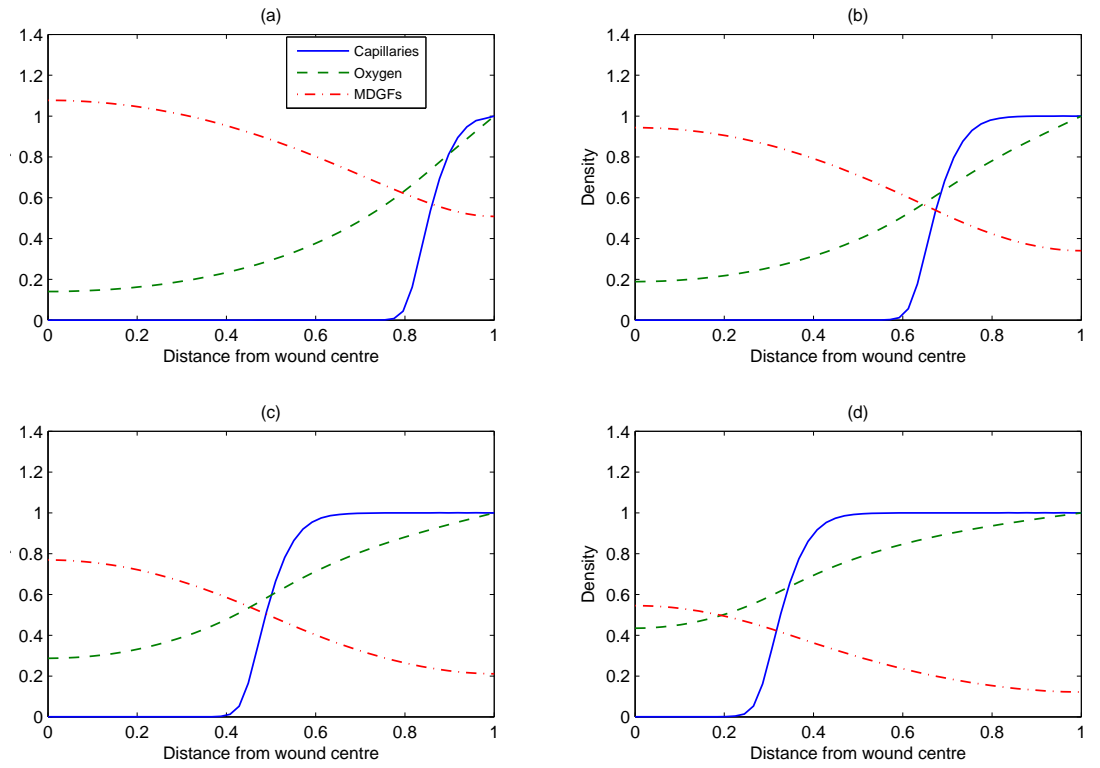

Figure 1: Numerical simulation of healing for a non-contaminated wound. The progression of capillary density at times $t=15,25,50$ and 75 is given in $(a),(b),(c)$ and $(d)$ respectively. Parameter values are: $d_{1}=10^{-3}, d_{2}=1, d_{3}=0.05, d_{4}=10^{-3}, a_{1}=0.5, a_{2}=10, a_{3}=2.5$, $a_{4}=7.5, a_{5}=0.75, a_{6}=0.25, a_{7}=0.5, a_{8}=0$
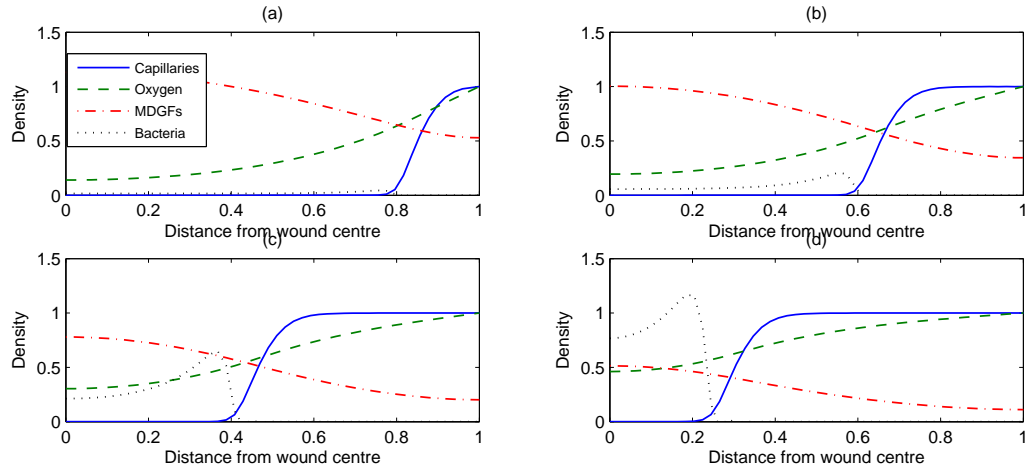

Figure 2: Numerical simulation of healing for a wound contaminated with a low density nonvirulent bacteria. The progression of capillary density at times $t=15,25,50$ and 75 is given in $(a),(b),(c)$ and $(d)$ respectively. Parameter values are as in Figure 1 with the exceptions: $a_{8}=0.05, a_{9}=0.05$ 

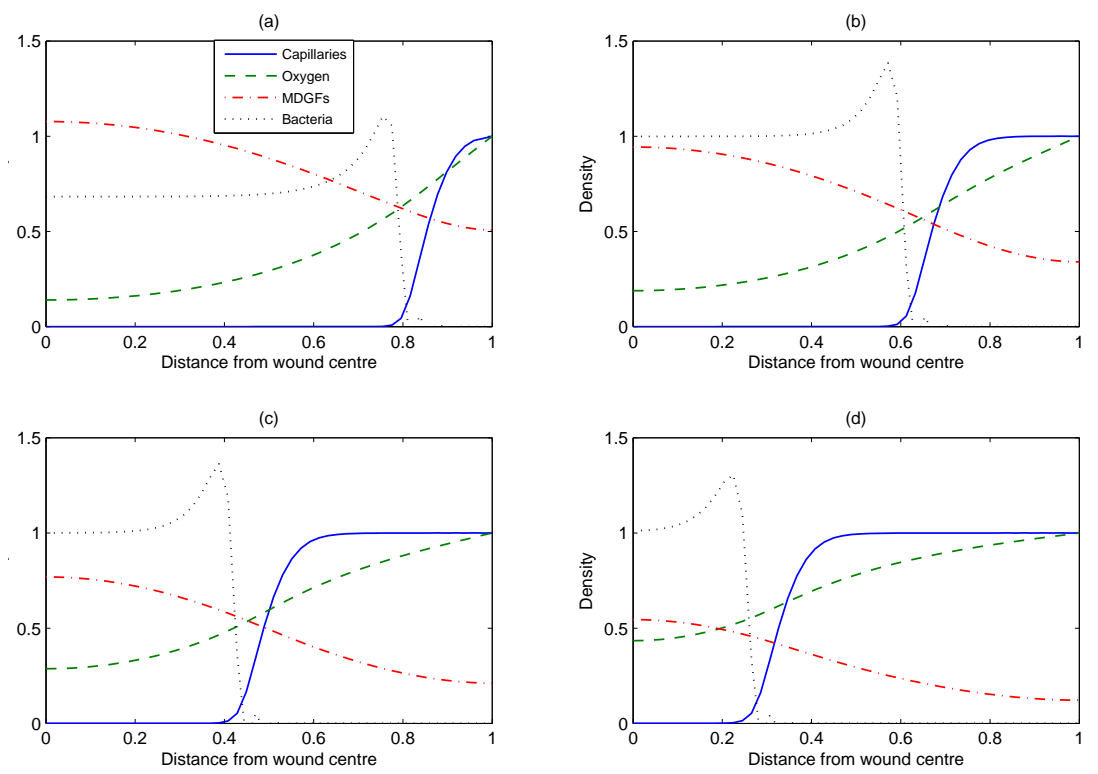

Figure 3: Numerical simulation of healing for a wound contaminated with a high density nonvirulent bacteria. The progression of capillary density at times $t=15,25,50$ and 75 is given in $(a),(b),(c)$ and $(d)$ respectively. Parameter values are as in Figure 1 with the exceptions: $a_{8}=0.05, a_{9}=0.25$

the wound is similar to that of a non-contaminated wound. This accords with the fact that a low level of harmless bacteria in a wound enhances the rate at which it heals [9].

The results in Figure 3 are for a wound containing a high density of harmless bacteria. The progression of capillary density is very similar to the progression we observed in the simulation of the previous wound. This indicates that the wound is healing in a regular manner. This is in contrast to the assertion that there exists a bacteria density threshold beyond which a wound becomes infected. Our results therefore suggest that the number of bacteria in a wound do not determine whether a wound becomes infected.

The third case we considered involves wounds that are contaminated with virulent bacteria. As we did in the second case, we present results for a wound whose bacteria density is low and one with a high bacteria density. The results shown in Figure 4 illustrate a wound with a low bacteria density. We observe that early in the process, the wound appears to heal normally and then stalls as time progresses. The virulence of the bacteria has delayed the healing process. We can therefore say that the wound is infected. Next, Figure 5 presents results for a high density virulent bacteria. Observe that capillaries have failed to propagate across the wound space from the very onset of the healing process. The later result illustrate the classic condition of chronic infection. In both cases the results support the assertion that bacterial virulence determines whether a wound becomes infected. 

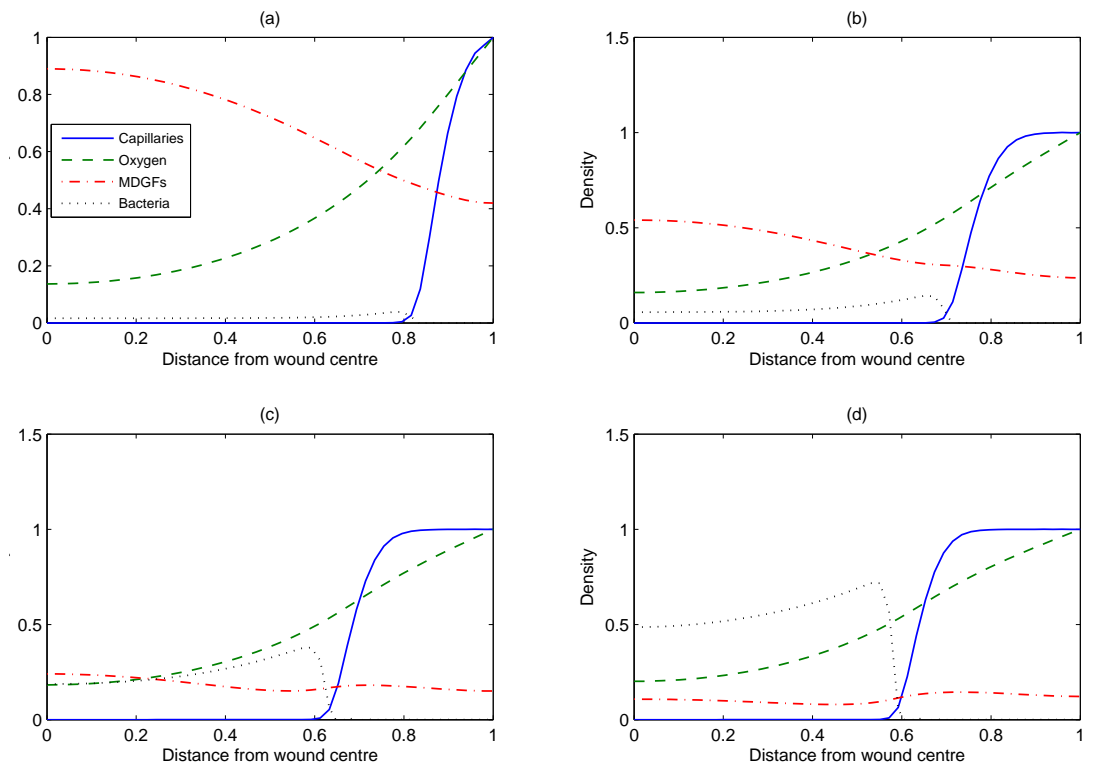

Figure 4: Numerical simulation of delayed healing for an infected wound with low bacteria density. The progression of capillary density at times $t=15,25,50$ and 75 is given in $(a),(b),(c)$ and $(d)$ respectively. Parameter values are as in figure 1 with the exceptions: $a_{8}=10, a_{9}=0.05$
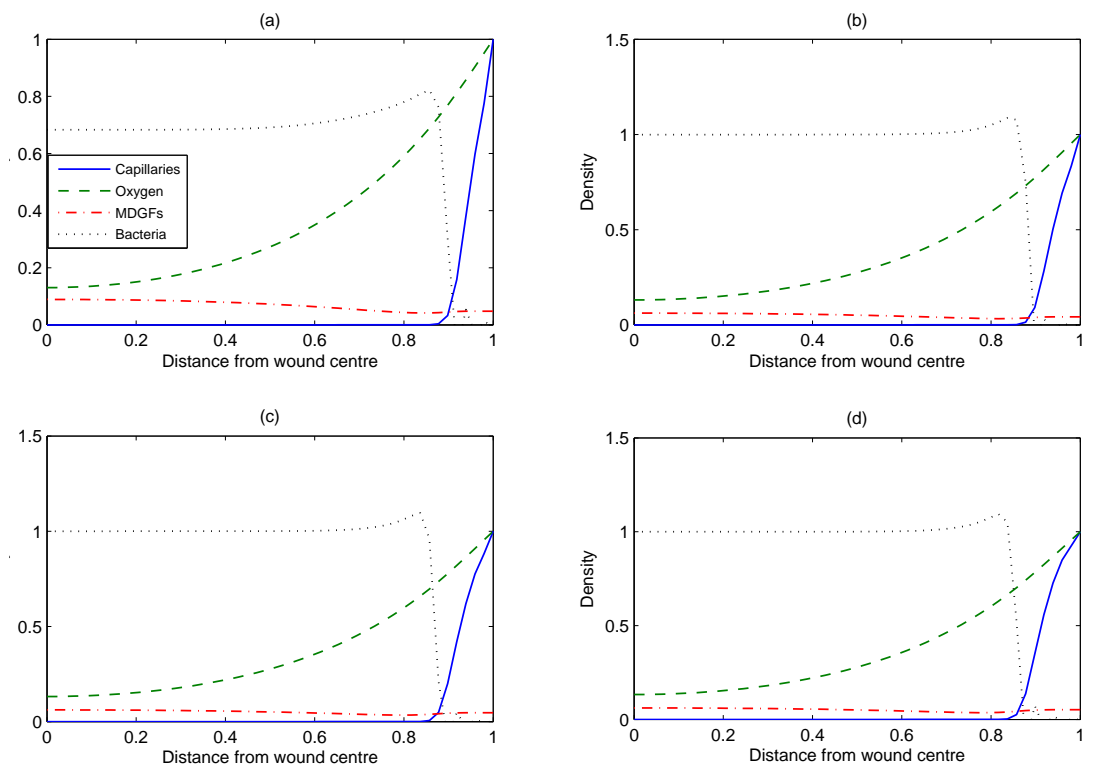

Figure 5: Numerical simulation of a chronic infected containing a high bacteria density. The progression of capillary density at times $t=15,25,50$ and 75 is given in $(a),(b),(c)$ and $(d)$ respectively. Parameter values are as in Figure 1 with the exceptions: $a_{8}=10, a_{9}=0.25$ 


\section{References}

[1] F. Arnold, D. C. West. Angiogenesis in wound healing. Pharmac. Ther. 52 (1992), 407-422.

[2] P.G. Bowler. The $10^{5}$ bacterial growth guideline: reassessing its clinical relevance in wound healing. Ostomy/Wound Manage 49 (2003),44-53.

[3] W.S. Bullough. Cell Replacement after Tissue Damage in C. Illingworth (Ed.), Wound Healing, Churchill, London 1966.

[4] A.Q. Cai, K.A. Landman, B.D. Hughes. Multi-scale modeling of a wound-healing cell migration assay. J. Theoret. Biol. 245 (2007) no. 3, 576-594.

[5] R.A.F. Clark. Overview and general considerations of wound repair. In: The Molecular and Cellular Biology of Wound Repair (R. A. F. Clark and P. M. Hendson, eds) 3-34, Plenum, New York 1988.

[6] R.A.F Clark. Wound repair. Curr. Opin. Cell Biol. 1 (1989), 1000-1008.

[7] R.A.F Clark. Growth factors and wound repair. J. Cell. Biochem. 46 (1991), 1-2.

[8] R.A.F Clark. Regulation of fibroplasia in cutaneous wound repair. Am. J. Med. Sci. 306 (1993), 42-48.

[9] R. Edwards, K.G. Harding. Bacteria and wound healing. Current Opinion in Infectious Diseases: 17(2004), no. 2, 91-96.

[10] S. Enoch, K. Harding. Wound bed preparation: the science behind the removal of barriers to healing. Wounds 15 (2003), 213-229.

[11] E.A. Gaffney, K. Pugh, P.K. Maini, F. Arnold. Investigating a simple model of cutaneous wound healing angiogenesis. J. Math. Biol. 45 (2002), no. 4, 337-374.

[12] D. Hilhorst, J.R. King, M. Rger. Travelling-wave analysis of a model describing tissue degradation by bacteria. European J. Appl. Math. 18 (2007), no. 5, 583-605.

[13] D. Hilhorst, J.R. King, M. Rger. Mathematical analysis of a model describing the invasion of bacteria in burn wounds. Nonlinear Anal. 66 (2007), no. 5, 1118-1140.

[14] J.R. King, A.J. Kooerber. Modeling host tissue degradation by extracelluar bacterial pathogens. Mathematical Medicine and Biology 20(2003), 227-260.

[15] A.J. Koerber, J.R. Kingand, P. Williams. Deterministic and stochastic modelling of endosome escape by Staphylococcus aureus: "quorum" sensing by a single bacterium. J. Math. Biol. 50 (2005), no. 4, 440-488. 
[16] A.J. Koerber, J.R. King, J.P. Ward, P. Williams, J.M. Croft, R.E. Sockett. A mathematical model of partial-thickness burn-wound infection by Pseudomonas aeruginosa: quorum sensing and the build-up to invasion. Bulletin of mathematical biology. 64 (2002), no. 2:239-59.

[17] S.A. Maggelakis, A.E. Savakis. Modeling techniques in epidermal wound healing. Computational methods in biophysics, biomaterials, biotechnology and medical systems: algorithm development, mathematical analysis, and diagnostics, 2 (2003), 91-118, Kluwer Acad. Publ., Boston, MA.

[18] S.A Maggelakis. A mathematical model of tissue replacement during epidermal wound healing. Applied Mathematical modelling 27 (2003), 189-196.

[19] S.A Maggelakis. Modeling the role of angiogenesis in epidermal wound healing. Discrete and Continuous Dynmical Systems Series B 4 (2004), 267-273.

[20] S. McDougall, J. Dallon, J. Sherratt, P. Maini. Fibroblast migration and collagen deposition during dermal wound healing: mathematical modelling and clinical implications. Philos. Trans. R. Soc. Lond. Ser. A Math. Phys. Eng. Sci. 364 (2006), no. 1843, 1385-1405.

[21] G. Odland, R. Ross. Human wound repair: I. Epidermal regeneration. J. Cell Biol. 39 (1968), 135-151.

[22] G.J. Pettet, H.M. Byrne, D. L. McElwain, J. Norbury. A model of wound-healing angiogenesis in soft tissue. Mathematical biosciences. 136 (1996), no. 1, 35-63.

[23] G.J. Pettet, A.J. Chaplain, D.L.S. McElwain, H.M. Byrne. On the role of angiogenesis in wound healing. Proc. R. Soc. Lond. B 263 (1996), 1487-1493.

[24] P.J. Polverini, P.S. Cotran, M.A. Gimbrone, E.R. Unanue. Activated macrophages induce vascular proliferation. Nature. 269 (1977), 804-806.

[25] M.C. Robson, B.D. Stenberg, J.P. Heggers. Wound healing alterations caused by infections. Clin. Plast. Surg. 17 (1990), 485-492.

[26] R. Rudolph, J. Vande Berg, H.P. Egrlich. Wound contraction and scar contracture. In: Wound Healing: Biochemical and Clinical Aspects (I. K. Cohen, R. F. Diegelmann and W. J. Lindblad. eds) Saunders, Philadelphia (1992), 96-114.

[27] J.A. Sherratt, J.D. Murray. Models of epidermal wound healing. Proc. R. Soc. Lond. B 241 (1990), 29-36.

[28] J.A. Sherratt, J.D. Murray. Mathematical analysis of a basic model of epidermal wound healing. J. Math Biol. 29 (1991), 389-404.

[29] M.J. Tindall, S.L. Porter, P.K. Maini, G. Gaglia, J.P. Armitage. Overview of mathematical approaches used to model bacterial chemotaxis. I. The single cell. Bull. Math. Biol. 70 (2008), no. $6,1525-1569$. 
[30] M.J. Tindall, P.K. Maini, S.L. Porter, J.P. Armitage. Overview of mathematical approaches used to model bacterial chemotaxis. II. Bacterial populations. Bull. Math. Biol. 70 (2008), no. $6,1570-1607$.

[31] H.V. Waugh, J.A. Sherratt. Macrophage dynamics in diabetic wound healing. Bull. Math. Biol. 68 (2006) no. 1, 197-207. 